\title{
Norton's material theory of analogy
}

\author{
Paul Bartha \\ Department of Philosophy, University of British Columbia, Canada
}

\section{A B S T R A C T}

In his book, The Material Theory of Induction, Norton argues that the quest for a universal formal theory or 'schema' for analogical inference should be abandoned. In its place, he offers the "material theory of analogy": each analogical inference is "powered" by a local fact of analogy rather than by any formal schema. His minimalist model promises a straightforward, fact-based approach to the evaluation and justification of analogical inferences. This paper argues that although the rejection of universal schemas is justified, Norton's positive theory is limited in scope: it works well only for a restricted class of analogical inferences. Both facts and quasi-formal criteria have roles to play in a theory of analogical reasoning.

\section{Introduction}

John Norton devotes one chapter of his book, The Material theory of Induction, to a discussion of analogical inference. ${ }^{1}$ As Norton recognizes, this "venerable form of inductive inference" has had spectacular successes (and failures) in mathematics, physics, biology and other fields. Starting with Aristotle, philosophers have acknowledged the importance of analogies in their discussions of inductive inference, but their attempts to characterize the logic of analogical reasoning have been rather unsuccessful.

Norton believes that the "material" approach offers a promising alternative, and he provides a straightforward two-part analysis. First, in line with the main thesis of his book, Norton makes a negative claim: formal analyses, approaches that characterize and appraise analogical reasoning in terms of conformity to some schema or rule, are hopeless and should be abandoned. Second, Norton proposes a positive alternative: his "material theory" of analogical inference. Analogies are "factual matters to be explored empirically" (Norton, 2018, p. 117). ${ }^{2}$ Analogical inferences are used to expand or refine an existing analogy between two systems. They derive their "warrant" from local facts rather than from formal rules or principles. Each analogical inference is "powered" or "driven" by a set of material facts that embraces the two systems. Norton calls this the fact of analogy. ${ }^{3}$ The argument succeeds if the fact of analogy, together with some additional observations, provides a warrant for the conclusion. Norton illustrates and supports his analysis with three detailed examples.

The negative claim reflects Norton's views about inductive inference in general. Those views are summarized in two slogans: "All induction is local" and "No universal rules on induction" (2). But Norton's positive theory of analogy differs in one important respect from his positive accounts of other types of inductive inference. Although Norton rejects the idea of a universal inductive logic, he is fine with local inductive logics:

A logic of induction is applicable in some domain if the facts of that domain match the factual restrictions of the logic of induction. Since there is no universally applicable factual restriction, in general, different domains require different inductive logics (322).

For example, Bayesian reasoning and probabilistic principles can play this role:

There are many domains in which varieties of Bayesian analysis are authorized and can be applied. (324).

The existence of domain-specific logics is the farthest that Norton goes in finding a compromise with formal epistemology. We find no such compromise, however, when it comes to analogical inference. Indeed, Norton contrasts the "formal precision" of Bayesianism with analogical reasoning which "struggle[s] to separate the strong from the weak analogies" (324). Order is not to be found: "there will be at best a loose similarity only between different analogical inferences in that, in all of them, we are authorized to pass properties from one system to another" (127). Norton thus leaves no room even for "local" formal guidelines. Call this position extreme materialism about analogical inference.

This paper is an examination of Norton's theory of analogical

E-mail address: paul.bartha@ubc.ca.

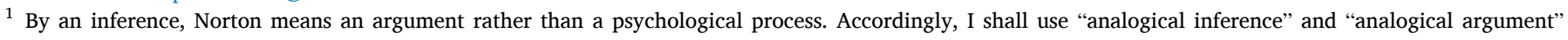
interchangeably.

2 All page references to (Norton, 2018) are to the posted version of June 26, 2018.

3 The term is somewhat misleading, since the fact of analogy is typically a conjecture. Norton tells us that it involves "inductive risk" (128). 
inference. His account has a number of attractive features. Norton is surely right that there is no universal formal schema or logic of analogical reasoning. His material theory of analogy directs us towards close "empirical investigations" of the facts, and away from the formulation and refinement of "formal principles" for analogical reasoning (129). Arguably, the orientation towards specific facts agrees with scientific practice when it comes to dealing with analogies. Another apparent virtue of the material theory is that it solves, or perhaps dissolves, the problem of providing a justification for analogical reasoning, once again by stressing a sober focus on the facts of the particular argument rather than on lofty philosophical objectives. Above all, as Norton himself notes, his account has the appeal of simplicity. The material theory promises fidelity to scientific practice, a contextsensitive treatment of individual analogies, and the relief of dispensing with will-o'-the-wisp theories that bear little resemblance to actual scientific reasoning.

Despite these virtues, I believe that Norton's negative position, the complete rejection of theories that include formal criteria for analogical reasoning, is a mistake. My principal criticism is that Norton holds such theories to an unrealistically high standard. There is plenty of middle ground between abstract schemas and extreme materialism.

I have two main criticisms of Norton's positive account. The first, and most important, is that this account has limited scope. The material theory works very well for some analogical arguments, but it gets the analysis wrong for many others. What Norton calls the fact of analogy is commonly the end-point rather than the driver of analogical inference. The material theory thus excludes many perfectly respectable analogical arguments. Secondly, as a consequence of its limited scope, Norton's analysis provides only limited insight into the justification of analogical reasoning.

My overall claim, then, is that both extremes-abstract formal logics of analogy and Norton's material theory-are unsatisfactory. In the final part of the paper, I argue that there is room in the middle for what I call a quasi-formal theory of analogy: a theory that uses formal apparatus but makes no claim to be a logic. Since it is Norton's main target, my focus will be on my own "articulation model" (Bartha, 2010); however, I also devote some attention to field-specific accounts of analogical reasoning.

The paper proceeds as follows. Section 2 outlines the desiderata for any theory of analogy. Section 3 presents and evaluates Norton's negative analysis. Section 4 describes Norton's positive account, the material theory of analogy. Section 5, the heart of the paper, develops my main criticisms. The final two sections are devoted to finding middle ground. Section 6 compares Norton's material theory to the articulation model on providing guidance for analogical reasoning. Section 7 contrasts how the two theories explain the justification for analogical arguments.

\section{What do we want from a theory of analogy?}

An analogical argument is commonly understood as an explicit inference that cites accepted similarities between two objects or systems of objects (also called the source domain and the target domain) to support the plausible conclusion that some further similarity exists. The focus here is practical: we use analogies to decide which conjectures are worth exploring. We want to know which analogical arguments are plausible. A theory of analogy (and analogical inference) tells us how such arguments work, or how they should work. A crucial first step is to spell out what we want from such a theory. We need to specify the key objectives and the standard of precision.

As a preliminary point, we can distinguish between descriptive and normative goals for a theory of analogical reasoning. There is one principal descriptive goal:

Understanding. Provide a faithful and insightful representation of the pattern (or patterns) of analogical reasoning employed by scientists. $^{4}$

The principal normative goal is to provide useful guidance in evaluating analogical arguments. Bartha (2010, 2019) identifies three normative objectives for a theory of analogy, each of which can be formulated with different levels of precision:

Criteria. Provide criteria for evaluating analogical arguments; more liberally, provide guidance for analogical reasoning. These criteria are understood to be fallible: good analogical arguments may turn out to have false conclusions.

Justification. Provide a philosophical justification for analogical reasoning; more liberally, explain how individual analogical arguments justify their conclusions.

Confirmation. Explain the role of analogies in theoretical confirmation; more liberally, accommodate analogical reasoning into a broad context that includes other types of inductive inference.

One way to approach these normative objectives is to develop a formal theory of analogy in the tradition of inductive logic. This approach is exemplified by Carnap (1980), early work by Hesse (1964), and others (Kuipers, 1988; Niiniluoto, 1988; Romeijn, 2006). A recent example is (Dardashti, Thebault, \& Winsberg, 2017; Dardashti, Hartmann, Thebault \& Winsberg 2019), which uses Bayesian networks to model analogical confirmation. Hesse (1966) offers a philosophical model that is less formal than the Carnapian approach, but still explicitly addresses each of the above objectives.

I believe that Norton intends his material theory to serve as both a descriptive and normative model of analogical reasoning. The former is evident, since Norton bases his account on the idea that "the scientific literature approaches analogies as factual matters to be explored empirically" (117). On the normative side, I submit that Norton accepts liberalized versions of at least the first two tasks for his own theory of analogy.

Consider the first point: a theory of analogical reasoning ought to provide guidance. If we interpret this objective as a demand for formal criteria, Norton is disparaging:

Reasoning by analogy ... has been the subject of persistent analysis from the perspective of formal approaches to inductive inference. The goal has been to find the formal criteria that distinguish good from bad analogical inferences. These efforts have met with mixed success, at best. (116)

But Norton still accepts the broad idea that a theory of analogical reasoning should provide genuine guidance, since he claims that the material theory does exactly that. He writes that the material approach "reorients our focus" towards facts rather than formal principles (129), and he illustrates the point with detailed examples. The goal is still to identify good analogical inferences.

Consider the second point: a theory should help to explain the justification for analogical inferences. Norton notes the failure of past theories to solve this problem (123). He is unsympathetic to any justification of analogical inference as an argument form. But the need for justification of individual analogical arguments remains front and centre in Norton's account, and as always, he looks to facts rather than to

\footnotetext{
${ }^{4}$ Of course, analogical reasoning is widely used in non-scientific settings
such as legal reasoning, philosophical reasoning, and everyday problem-

${ }^{4}$ Of course, analogical reasoning is widely used in non-scientific settings
(such as legal reasoning, philosophical reasoning, and everyday problemsolving activities), but in this paper I will limit my attention to scientific uses of analogy.
} 
formal principles. Norton believes that the material theory is on much firmer ground than any formal theory when it comes to justification:

The material theory of induction succeeds in simplifying our understanding of analogical reasoning in its acceptance of the dual role of facts: they may be premises in arguments and they may also serve as warrants of inference. Crucially, the material theory allows that displaying such facts provides the justification of the analogical inference and is the endpoint of analysis that seeks to determine the validity of the analogical inference...

A formal approach faces a more elaborate challenge. It can allow that a fact of analogy can somehow play a role in justifying an analogical inference. But this recognition cannot terminate a successful formal analysis. The validity of an analogical inference must be established ultimately by displaying conformity with a universal schema. (1378)

Norton does not say much about analogies and confirmation, so we may set aside the third objective. In summary, I make the basic assumption, which I take to be shared by Norton, that in addition to delivering a model that is descriptively adequate, a satisfactory theory of analogical inference should meet the first two normative objectives identified above.

\section{Norton's negative account: rejection of formal theories}

Norton holds formal theories of analogy to the very high standard of providing an inductive logic. He identifies three salient requirements:

It is to be non-contextual, universal and formal. The numerical facts of arithmetic are non-contextual-that is, independent of the context. In abstracted form, they hold for eggs, acrobats and every other sort of individual. The rules are universal; they don't come with restrictions to particular domains. It is the same arithmetic for eggs and acrobats. And the rules are formal in the sense that they attend only to the form of the sentence asserting the data: six .... of 12 .... . The matter-eggs or acrobats—is ignored. (28)

A successful logic of analogy should provide non-contextual, universal formal schemas for good analogical inferences.

Norton argues (convincingly) that past theories fall well short of these requirements, and that future efforts to develop a "logic of analogy" may be expected to fail as well. He begins by considering one simple formal schema, drawn from an old logic text (Joyce, 1936) and similar to the account of Mill (1904), which he calls "bare analogy" (117):

$S_{1}$ is $P$.

$S_{2}$ resembles $S_{1}$ in being $M$.

[therefore] $\mathrm{S}_{2}$ is $\mathrm{P}$.

We might also call this an argument from similarity. Although Norton recognizes that this argument form has been "quite fertile in the history of science" (118), he notes that it faces obvious counterexamples, that it needs to be embellished (typically by adding conditions about the connection between $\mathrm{M}$ and $\mathrm{P}$ ), and that even the embellished versions frequently yield false conclusions. He sums up:

It is a good tonic, therefore, to recall what successful rules look like in deductive logic. Modus ponens is a valid inference, always. Affirming the consequent is a deductive fallacy, always. We should take this as a warning. That our rules [for analogical reasoning] need to be protected by vagueness and ambiguity may be an alert that there is no precise rule to be found. (120-1)

Formal schemas for analogical reasoning are inevitably vague, incomplete and highly fallible; accordingly, they fail to be universal and non- contextual. So, they fail as a logic.

Norton directs most of his discussion and criticism towards quasiformal theories of analogy, specifically the theories developed by Hesse (1966) and me (Bartha, 2010). Both accounts make use of a tabular representation in which analogical inferences have both a vertical and a horizontal dimension. Here is the representation (Bartha, 2010, p. 15):

The diagram represents both the positive analogy (similarities) and negative analogy (differences) between the two domains, as well as the analogical conclusion in which a further similarity $\left(Q^{*}\right)$ is inferred as plausible. The horizontal relationships in this diagram are similarities and differences between the two domains. The vertical relationships are always causal relations in Hesse's work, but I (Bartha, 2010) expand this idea to include predictive, explanatory, functional and statistical relations. As Norton notes, the basic idea in Bartha (2010) is that an analogical inference is good if (i) there is a "prior association": a clear articulation of the vertical relationship in the source domain, and (ii) there is a "potential for generalization", i.e., no obstacle to a generalization that extends this relationship to the target domain. (The key issue is whether or not the features that belong to the negative analogy play an essential part in the prior association, potentially blocking transfer to the target.) These two broad principles are unpacked in different ways to yield criteria for evaluating analogical arguments, depending on the type of vertical relation. Norton comments that these accounts "significantly enrich the original formal notion of bare analogy" and writes: "if a formal analysis of analogical inference can succeed, this is likely the right direction" (125).

Naturally, Norton denies both the antecedent and the consequent. In order to understand his objections to Hesse and Bartha, it is vital to keep in mind that Norton premises his critiques on the assumption that both accounts aim to provide a formal logic of analogy. For instance, Norton characterizes the tabular representation in Fig. 1 as a "general schema" (121). He extracts a more elaborate "general schema" for good analogical reasoning from Hesse's work (123). One of his principal objections to the articulation model in (Bartha, 2010) is that despite its complexity, it still fails to deliver "a fully elaborated formal schema" (126). He goes on to develop a more specific objection to the articulation model: "if it is to give a formal schema for analogical inference", then it faces a daunting regress problem: not only must it provide a high-level formal schema, but also "schemas for each of the lower level forms of inductive inference" corresponding to the different types of vertical relation (127).

In short, Norton sees the quasi-formal theories of Hesse and Bartha as ever-expanding top-down elaborations of "bare analogy" that attempt, but fail, to close the gap between the formal schema and real-life arguments. His main objection is nicely summed up as follows:

Their embellishments are never quite embellished enough. There is always some part of the analysis that must be handled intuitively without guidance from strict formal rules. (116)

And again:

Efforts to narrow the gap between the schema and the cases will require the proposal of more elaborate, more fragmented schemas ... No matter how complicated the successive proposals become, they will still never be adequate to all the cases. Gaps will remain. (128)

I think that Norton is right. Neither the articulation model nor any model on offer meets the high standards that Norton sets for inductive logic. At this point, however, we should ask whether Hesse or Bartha ever intended to offer a logic of analogy. The answer is plainly "no". 5 Hesse's later work (1974) makes it clear that her understanding of logic is more liberal than Norton's. Bartha (2010) seems to be on the same

\footnotetext{
5 Each, admittedly, occasionally uses the phrase "logic of analogy". See Bartha, 2010, p. 105; Hesse (1966) has a chapter titled "The Logic of Analogy".
} 


\begin{tabular}{lll} 
Source & Target & \\
$\mathrm{P}$ & $\mathrm{P}^{*}$ & (positive analogy) \\
$\mathrm{A}$ & $\sim \mathrm{A}^{*}$ & (negative \\
$\sim \mathrm{B}$ & $\mathrm{B}^{*}$ & analogy) \\
\hline $\mathrm{Q}$ & $\mathrm{Q}^{*}$ & (plausibly)
\end{tabular}

Fig. 1. Tabular representation of analogical reasoning

side as Norton when it comes to formal schemas: "the hope for a single, simple rule of analogical inference is likely to be futile" $(2010,23)$. As to Norton's regress problem and the need for explicit lower-level schemas, I noted that I can at best offer "partial validation" of the articulation model that "takes for granted certain models of explanation, causation, and so forth" $(2010,30)$. I also introduced an explicitly rhetorical and contextual framing device: an analogical inference is "presented by an enthusiastic advocate to a polite but moderately skeptical interlocutor, the critic" $(2010,5)$. This device of advocate and critic is useful to "help set a standard of justification that can be varied to reflect the demands of different settings" $(2010,5)$, and is regularly invoked in the development of the articulation model. All of these points show that the articulation model was never intended to serve as an inductive logic.

What happens if we drop the assumption that Hesse's and Bartha's theories are meant to be formal logics of analogy? Norton's objection about failure to meet standards of universality, formality and contextindependence disappears. Norton's regress objection to Bartha's articulation model also loses force. But now Norton might take a different line: what then is the purpose of the formal schema, or formal criteria, put forward by these theories? If the formal apparatus does not establish precise rules for the assessment of analogical arguments, and does not provide justification for accepting arguments that "fit" the inference rules (and rejecting those that do not), then what purpose does it serve? I'll return to this question in the final sections of the paper. Here I offer a partial answer. A quasi-formal theory can fall short of the requirements for an inductive logic, yet still help with the tasks identified in section 2 .

It is helpful, at this point, to make an important clarification about the precision of Norton's own theory of analogy. There is a sense in which Norton's material theory retains very high standards of precision for the assessment of analogical arguments. As I shall show in the next section, Norton believes that in evaluating an analogical argument, scientists in effect construct what I shall call a micro-logic, an inductive logic specific to an inferential context. This micro-logic both drives and warrants the analogical inference. We must, however, distinguish between the multiple micro-logics and Norton's material theory, which is not itself a logic and is not the sum of the micro-logics. Norton provides no algorithm or rule for generating the micro-logics; in no way does the material theory imply or contain them. In short, the material theory is an informal model of analogical inference, on the same footing as accounts like the articulation model.

The main point, then, is that we need to adopt a consistent standard if we are to compare Norton's theory fairly to other theories of analogy in terms of meeting the objectives identified in section 2. If we insist on the precision and universality of logic, then Norton's theory of analogy fails as badly as the other accounts. If we lower the standard to allow informal and imprecise guidelines, then we should compare theories based on a liberal reading of the two key normative objectives: how well they provide guidance, and how well they explain the justification of analogical arguments. We should also compare how well they function as descriptive models, i.e., how well they conform to actual scientific practice. That is what I will do (sections 6 and 7) after outlining and critiquing Norton's positive theory (sections 4 and 5).

\section{Norton's positive account: the material theory of analogy}

The purpose of Norton's material theory is to re-orient our attention away from universal schemas and formal criteria. Justification for an analogical inference derives from material facts. Although Norton's theory appears straightforward, a few points deserve elaboration. In particular, I shall argue that his theory imposes some important restrictions on allowable forms of analogical inference.

According to Norton, the material analysis of an analogical argument involves two steps:

i) Step 1: Identify the fact of analogy. Norton describes this as "a factual state of affairs that arises when two systems' properties are similar, with the exact mode of correspondence expressed as part of the fact" (128). It is simply "some fact that embraces both systems" (128). The fact of analogy is a "local" property shared by the two systems, and is something "to be explored empirically" (117). It is important to clarify that the fact of analogy is typically a conjecture, so that this step involves "inductive risk" (128).

ii) Step 2: Make the analogical inference. The fact of analogy plus additional factual properties of the target system warrant the analogical inference. Norton's exact phrase is "the analogical inference warranted by a fact of analogy" (128).

The terminology here may be a little confusing, since "analogical inference" can refer either to a specific step (step 2) or to the full inference (steps 1 and 2 together). I shall generally reserve "analogical inference" and "analogical argument" for the entire inference; I use "step 1 " and "step 2" for the two parts.

The best way to understand Norton's ideas is by considering his first and simplest example: Galileo's use of analogy to infer the existence of mountains and valleys on the moon. In his 1610 work, Siderius Nuncius (The Starry Messenger), Galileo recorded his observations, through the telescope, of the advancing edge of sunlight on the moon. In particular, he noticed bright points of light appearing ahead of the advancing edge and eventually joining up with it. He noted the analogy to the illumination of mountains on earth: "before the rising of the sun, are not the highest peaks of the mountains illuminated by the sun's rays while the plains below remain in shadow?" $(1610,33)$. His conclusion was that the bright points mark the locations of mountains on the moon, and he even provided a reasonably good estimate of the height of the mountains. In a similar way, he argued that certain dark patches on the moon indicate depressions or valleys.

Norton argues that the material theory provides an excellent analysis of Galileo's reasoning. Here is how he describes the fact of analogy:

The mode of creation of shadows on earth and of the moving dark patterns on the moon is the same: they are shadows formed by straight rays of sunlight. (131)

The "additional factual properties" of the target are that there are points of light ahead of the advancing edge of sunlight, and that there are dark patches that do not change over time. The analogical inference to the existence of mountains and valleys follows from this decomposition: the bright spots must be elevated above the surface of the moon, and the dark patches must be depressions. As Norton stresses, the reasoning diverges from "bare analogy". The conclusion is not based on a list of similarities per se, but on laws of optics common to earth and moon. Norton also notes that an element of inductive risk is present in accepting the fact of analogy: other mechanisms might be responsible for producing light and shadow on the moon (132). 
$S$ (source domain)

$T$ (target domain)

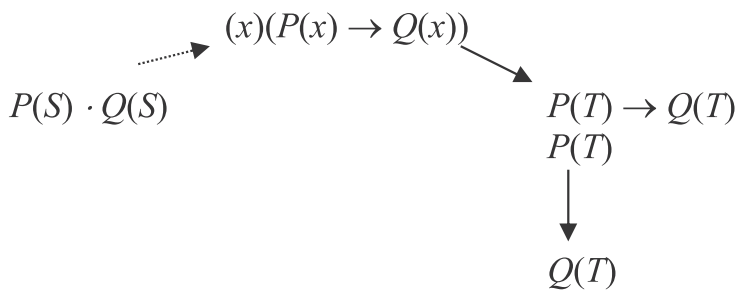

Fig. 2. Argument from example.

The central concept of the material theory is clearly the fact of analogy. Although Norton does not say so, I suggest that the fact of analogy is best understood as a low-level generalization broad enough to cover both domains. That certainly fits well with remarks that Norton makes about the Galileo example:

The earth functions as a convenient surrogate for any uneven body turning under unidirectional light. (131)

The inference is not driven as much by analogy as by subsumption of the moon into a larger class of illuminated bodies. (132)

Understood in this way, the fact of analogy is perfectly suited to serve as a proxy that allows us to dispense with any formal inductive schema: it both drives and justifies the inference. We may think of it as defining a micro-logic for the two domains. That is, we have a low-level schema that uses the fact of analogy, in combination with additional facts about the target domain, as warrant for the analogical conclusion.

Indeed, in important respects, Norton's analysis conforms to a pattern of reasoning that Aristotle (Aristotle, 1984) called the argument from example or paradeigma (Rhetoric 1402b15; Prior Analytics 69a1). Schematically, the pattern looks like this:

In Aristotle's example, $P$ is making war against neighbours, $Q$ is evil, the source domain $S$ is a known case (the war between Phocians and Thebans), and the target domain $T$ is a new case (war between Athenians and Thebans). The conclusion is that war between Athenians and Thebans is evil. The key elements of Norton's material theory are all present in the paradeigma argument.

The argument is not justified via conformity to any general schema for analogical reasoning. Instead, the low-level generalization (the fact of analogy) drives the inference.

The strength of the analogical argument is determined by the two steps in the inference.

Step 1 identifies and justifies the fact of analogy. It is an inductive step (indicated by the dotted arrow) because, as in the Galileo case, the fact of analogy is a conjecture based (in whole or in part) on our knowledge of the source domain.

Step 2 is what Norton calls the analogical inference warranted by the fact of analogy. It is a deductive inference (indicated by the solid arrow).

Norton's material theory directs us to strengthen an analogical inference through empirical investigation and refinement of the fact of analogy. The same point applies to the paradeigma argument pattern.

There are three main differences between Norton's material theory and the paradeigma. The first is that the fact of analogy always takes the particularly simple form $(x)(P(x) \rightarrow Q(x))$ for Aristotle but can be more complex for Norton. We encounter two principal forms within the material theory.

Form 1: the fact of analogy is a generalization.
This form appears in the argument from example, as illustrated in Fig. 2. In the Galileo case, the fact of analogy takes the form of a generalization involving the laws of optics. The domain of quantification is restricted (though broad enough to cover both earth and moon). In such cases, step 2 is a deductive inference.

\section{Form 2: the fact of analogy is a structural equation or determi- nation rule.}

Norton offers two additional examples, the Reynolds analogy and the liquid drop analogy. In these examples, the fact of analogy states a correlation between the values of variables in the source and target domains. On my reading, this means that the fact of analogy can be formulated as a structural equation (Pearl, 2009) or determination rule (Davies \& Russell, 1987; Russell, 1986). The overall analogical argument still conforms to the pattern of Fig. 2, but the logical form of the fact of analogy is a little more complex.

As a simple illustration of Form 2, consider an example adapted from (Davies \& Russell, 1987). Suppose that the dollar value that I assign to a used car is determined by its age, make, mileage, condition, color, and accident history. Symbolically, we can write this as:

$\mathrm{A}, \mathrm{M}_{1}, \mathrm{M}_{2}, \mathrm{C}_{1}, \mathrm{C}_{2}, \mathrm{H} \rightarrow \mathrm{V}$

The equation may, but need not, specify the exact form of functional dependence. We only need to know that any two cars that are 'the same' as regards these six parameters have equal value. Given the determination rule and the value of one car, I can infer by analogy the value of the other car. Step 2 of this analogical argument is once again a deductive inference, since the determination rule establishes a correlation between parameters in the two domains. The fact of analogy appears to take this form in Norton's discussion of the Reynolds analogy. ${ }^{6}$

While the above two forms have a central role in Norton's material theory, there are possible variations. Norton suggests that the fact of analogy might be understood vaguely or in probabilistic terms - most likely, as weakened versions of one of the above forms. The basic idea is clear: the fact of analogy is a low-level generalization or 'connection' covering both systems. It allows us to derive additional features of the target domain, either deductively (in the two forms identified here) or inductively. This gives us the second main difference between Norton's account and the paradeigma: the inference at step 2 may be either deductive (as in the Galileo case) or inductive (for probabilistic formulations).

The third difference is the justification, at step 1, for the fact of analogy. For Aristotle, it is generalization from a single case. For Norton, we have an inductive inference that draws on knowledge of the source domain but perhaps also on knowledge of the target and on additional background information. The exact nature of this inference will shortly take centre stage as we assess Norton's theory.

We may fairly conclude that Norton's theory of analogy is a generalization of the Aristotelian pattern. I will continue to refer to "step 1" (inductive inference to the fact of analogy) and "step 2" (Norton's "analogical inference", a conclusion about the target system) in my discussion of Norton's account. His examples are more intricate than Aristotle's example of making war on a neighbour. But intricacy does not change the basic structure of Norton's analysis: analogical reasoning reduces to two non-analogical inference steps. The analogical conclusion (step 2) must be derived from an intermediate low-level inductive generalization (step 1). There is nothing wrong with the Aristotelian

\footnotetext{
6 There is not enough space to permit discussion of this example. As for Norton's third example, the liquid drop analogy, the fact of analogy appears to be a combination involving both a restricted generalization and a determination rule.
} 
analysis, if it works. In the next section, however, I will argue that it does not work well for a range of cases.

\section{Assessment of Norton's positive theory}

\subsection{Limited scope: the fact of analogy is not always the driver}

This subsection develops my main criticism of Norton's material theory: it works well, but only for a restricted class of analogical inferences. As a result, Norton's theory has limited scope. It rules out entire categories of useful analogical inference.

It is important, first, to acknowledge the cases where Norton's theory works well. Norton tells us that "the fact of analogy must be able to power this [step 2] inference." Accordingly, Norton's examples have the following features:

(i) The fact of analogy (a relevant low-level uniformity) is readily identifiable. The fact of analogy must be identified with sufficient clarity to support the step 2 inference. This is the heart of Norton's analysis.

(ii) The fact of analogy has independent support. We require inductive support at step 1.

(iii) The fact of analogy is not the conclusion of the analogical argument. The fact of analogy is an intermediate step in deriving the analogical conclusion.

As we've seen, the theory works well for the Galileo example. I think that Norton's theory also functions well for the Reynolds analogy between processes that transfer momentum and heat. In this example and others like it, researchers use theory and experiment to establish criteria for when measured parameters in the source domain provide reliable information about the target. Sterrett (2006) notes that in some of these cases, we have a "governing equation" that describes one or both domains, while in others we have merely "the form of the equation in the most general sense, i.e., that the phenomenon is a function of quantity 1 , quantity 2 , etc." $(2006,76)$. These two cases correspond to the two possible forms for Norton's fact of analogy identified in section 4 .

I want to acknowledge a second point on which I agree with Norton. In nearly every case of an ultimately successful analogical inference (where the analogical conclusion turns out to be true), we do eventually find a fact of analogy that unifies the source and target domains. ${ }^{7}$ The pattern is familiar in mathematics: a successful analogy leads to the formulation of new concepts and ideas that enable us to see the source and target as special cases (Polya, 1954). We may say, then, that an ultimately successful analogical inference is "justified" or explained by a fleshed-out fact of analogy. However, Norton's concern (and mine) is how analogical arguments function at the early or intermediate stages of an inquiry. A theory of analogy should help us to determine whether an analogical argument is initially good, whether or not it turns out to be ultimately successful.

I now turn to my main objection to the material theory. Broadly speaking, if we set aside the "bare analogy" scheme of section 3, there are two contrasting models of the relationship between analogical arguments and generalization. There is a forward-directed picture: analogical reasoning proceeds from the source domain to an intermediate generalization, followed by application to the target. This is Norton's model. There is also a backward-directed picture: generalization guides analogical reasoning as an objective, rather than as an intermediate step. On this model, an analogical argument is constructed and evaluated by considering possible obstacles to the existence of some unifying generalization or fact of analogy. This pattern results in weaker

\footnotetext{
7 The qualification here arises because some have argued that purely formal analogical inferences have played an important role in physics (Fraser, 2018; Steiner, 1998). Arguably, there is no unifying fact of analogy in such cases.
}

but more flexible arguments than those Norton considers. In particular, we find cases of the following sorts:

a) No clear fact of analogy. There may be no specific identification of any fact of analogy.

b) No independent support for the fact of analogy. Even if a fact of analogy is identified, there may be no independent inductive argument offered in support, and there may be none available.

c) The fact of analogy is too vague to support any inference. The fact of analogy may be stated too vaguely to justify either a deductive or inductive inference to the analogical conclusion.

The key point is that there are cases of analogical reasoning where the fact of analogy operates backwards (as a goal) rather than forwards (as a driver). Let us consider a few examples.

(i) Mathematical analogies. (The fact of analogy is a remote conclusion.)

Mathematical analogies are commonly used to make conjectures and to help solve problems. Here are two simple examples (Bartha, 2010; Polya, 1954):

Triangles and tetrahedra: The three medians of a triangle have a common point of intersection. By analogy, we conjecture that the four medians of a tetrahedron have a common point of intersection. Rectangles and boxes: Of all rectangles with fixed perimeter, the square has maximum area. By analogy, we conjecture that of all rectangular boxes with fixed perimeter, the one with greatest volume is a cube.

Both arguments seem plausible. Yet in neither case does a local fact of analogy drive the inference. Specifically, there are two difficulties for Norton's approach. First: although we rightly suspect that there is a relevant generalization lurking in both cases - a general result that applies in two, three or any number of dimensions - we perceive the analogy, and perform the analogical reasoning, without invoking this generalization as an intermediate step. So these examples are a problem for the material theory as a descriptive account of how analogical reasoning actually proceeds. Second: the relevant generalizations emerge as a result of the analogical inferences rather than driving them. If we were to formulate the right generalization (the fact of analogy) in either case, we would immediately recognize that the analogical conclusion is a special case. It distorts the analogical argument to insist on the inductive generalization as an intermediate step. So the examples are also a problem for the material theory as a normative account of analogical reasoning; it is not even reasonable to reconstruct the arguments in a way that treats the fact of analogy as an intermediate step.

Norton's account has the order backwards for such cases. The fact of analogy is the ultimate result of analogical inference. Rather than an engine that pushes the reasoning forwards, it is a magnet that draws us towards an end point. ${ }^{8}$ This point applies as much to analogies in simple problem-solving exercises as to mathematical breakthroughs, as reflected in Poincare's remark that analogies in mathematics enable us "to perceive the possibility of generalization" $(1952,29)$. On Norton's account, we have no warrant for such analogical inferences.

(ii) Explanatory analogies. (The fact of analogy is the conclusion of the argument.)

In some explanatory analogies, the fact of analogy is best understood as the conclusion of the analogical inference-not as an intermediate

\footnotetext{
${ }^{8}$ It is important to remember, once again, that we are thinking of the warrant for inference at the early stage of inquiry.
} 
step. I proposed (Bartha, 2010, p. 96) the following structure for explanatory analogical arguments:

$Q$ explains $P$ in the source domain.

$Q^{*}$ (if true) would explain $P^{*}$ (analogue for $P$ ) in the target domain. Hence: $Q^{*}$ is plausible. ${ }^{9}$

My claim is that in some analogical arguments of this type, the analogical conclusion is, in effect, that features of the two domains might have explanations with a common structure. The fact of analogy is the conclusion of the argument.

As an illustration, consider the acoustical analogy, employed by a number of 19th century physicists attempting to explain the spectral lines of atomic hydrogen prior to the discovery of the Balmer formula. Stokes and Stoney suggested that spectral phenomena might be "analogous to the acoustical situation" of a tuning fork or vibrating string, which produces discrete frequencies (Maier, 1981, p. 51). They expected to find whole-number ratios between frequencies in the emission spectra. While this approach ultimately failed, many people initially regarded the conclusion as plausible.

Following Norton's analysis, we might try to identify the fact of analogy in this case with some conjectured set of properties common to tuning forks and emission spectra. The analogical conclusion (at step 2) will have to be something different - for example, the inference that we will find whole-number ratios between emission frequencies. The question is whether we can find a fact of analogy that plays the role that Norton assigns to it. Is there a conjectured fact about both domains, an intermediate principle that is independently supported and that drives us to the analogical conclusion? We might propose that the hypothesis that spectral phenomena behave like a tuning fork is the intermediate fact of analogy (step 1), and that we have a subsequent deductive inference that there are integral frequency ratios. If we decompose the argument in this fashion, then the fact of analogy requires independent inductive justification. But there is no inductive justification, independent of the analogy, for the conjecture that atomic spectra behave like tuning forks. So, on Norton's account, there is no warrant for the analogical inference.

The alternative view is that the hypothesis that spectral phenomena behave like a tuning fork, whether formulated vaguely or in precise terms, is really the conclusion of the analogical argument. The search for whole-number ratios between frequencies is one of a series of tests designed to determine whether that hypothesis is tenable, and what precise form it might take. The analogical argument remains viable until there is convincing evidence that we cannot put the frequencies in whole-number ratios.

It is instructive to compare this example to Galileo's reasoning about mountains on the moon - also an explanatory analogy. In the Galileo example, as Norton rightly argues, our knowledge of optics gives us independent inductive justification for the fact of analogy. For the acoustical analogy, we have no such independent justification. This is the case for many explanatory analogies: the fact of analogy is the terminus of the analogical inference rather than an intermediate step.

(iii) Predictive and explanatory analogies. (Fact of analogy is not identified, or too weak to power the "step 2" inference.)

One final problem for Norton is the existence of analogical arguments that proceed even when no fact of analogy is patent, or when the fact of analogy is too weak to support the "analogical conclusion" at step 2.

Consider the use of ethnographic analogy in archaeology. Currie (2016) describes how knowledge of contemporary and

\footnotetext{
${ }^{9}$ The argument form is distinct from inference to the best explanation. There is no assumption that $Q^{*}$ is the best explanation for $P^{*}$, and the conclusion is merely that $Q^{*}$ is a plausible hypothesis.
}

near-contemporary shamanistic practices among the San have been used to interpret motifs appearing on rock art. Among the San, the shamanistic motifs are used to symbolize both religious concepts and hallucinogenic experiences; the inference is that the very similar images appearing on rock art have a similar purpose. The analogy has been applied both to direct ancestors of the San (from 3000 years ago) and to older European cave art (20,000 years and older). The reasoning is very difficult to generalize. No fact of analogy emerges from the known cases to justify the "step 2" inference. A generalization such as "humans use certain motifs to symbolize religious concepts and hallucinogenic experiences" is overly broad, and it is unclear how to articulate a plausible weak version that would support the inference about European cave art. Nor does this seem to be what archaeologists do. To recast the argument in terms of a weak fact of analogy appears forced. There is debate about the role that qualified socio-cultural and environmental uniformities play in this type of inference, but the fact of analogy emerges as the result of the analogical inferences rather than as something prior.

To sum up: the material theory seems unable to account for inferences where the fact of analogy is the goal rather than the driver of the reasoning, or for inferences where the fact of analogy is not readily identified. The material theory must count such analogical inference as failures, exclude them from consideration, or force them into a mould in which an independently established fact of analogy is an intermediate reasoning step. By contrast, a backward-directed model of analogical reasoning is compatible with the lack of an explicitly formulated fact of analogy.

\subsection{Limitations on justification}

The second task of a theory of analogy is to provide philosophical justification for analogical reasoning. As we would expect, Norton has no interest in high-level philosophical justifications for analogical reasoning. His theory does provide a straightforward analysis of how justification goes for individual analogical arguments that fit the pattern of Fig. 2: inductive justification for the fact of analogy (step 1) along with justification (deductive or inductive) for the subsequent inference to a fact about the target domain (step 2). The decomposition, as we have seen, reduces the analogical argument to two non-analogical arguments.

As argued in section 5.1, the analysis simply doesn't work if the fact of analogy is not patent, not independently justifiable, or does not function as an intermediate step in the argument. A very different kind of justification is needed. In fact, once we move beyond Norton's model, in which one basic pattern of reasoning (illustrated by Fig. 2) applies across the board, we may need different guidelines for different types of analogical argument.

We may agree with Norton that looking for a universal schema or "logic" of analogy is a dead end. But we don't have to think of quasiformal criteria for analogical reasoning in this way. Instead, we can think of them as usefully identifying distinct patterns of analogical reasoning. I now turn to a defence of this role for quasi-formal theories of analogy.

\section{A practical role for quasi-formal theories: guidance}

If a formal or quasi-formal theory of analogy is not an inductive logic, then what purpose does it serve? Sections 2 and 3 offered a preliminary answer: it can help us to meet the basic objectives for any theory of analogy. In this section and the next, I elaborate by comparing the articulation model (Bartha, 2010) to Norton's theory. I focus specifically on how the two theories provide guidance about analogical reasoning, and how they account for the justification of analogical arguments. This section deals with the first objective. Section 7 compares the two theories in terms of the second objective.

The articulation model, as outlined earlier, is based on two broad principles: 
Prior association. There must be a clearly articulated causal, logical or explanatory relationship in the source domain.

Potential for generalization. There must be "no compelling reason" to deny that the prior association in the source domain could be generalized in a way that extends to the target domain.

The "articulation" aspect involves elaborating the requirement of prior association in terms of quasi-formal templates, and fleshing out the requirement of potential generalization in terms of distinct guidelines for different types of argument.

As we saw in section 3, Norton rejects this proliferation of "embellished schemas" for distinct types of analogical argument as both too complicated and as failing to "close the gap" between logic and scientific practice. As we saw in section 4, Norton needs no such distinctions because the material theory recognizes only one basic pattern of analogical reasoning, modeled on Aristotle's "argument from example". If the argument of section 5.1 is right, however, then Norton's picture is too simple. The articulation model handles analogical arguments in which the relationship between analogy and generalization is more indirect than in Norton's examples. The diversity of such arguments is the sole reason for distinguishing different types of analogical inference, and for attempting to formulate distinct guidelines.

Consider a typical mathematical analogy. The prior association is a mathematical proof in the source domain; the analogical conclusion is a result about the target domain; and the potential generalization (or fact of analogy), perhaps dimly glimpsed, is a mathematical result that subsumes both domains. Norton locates the generalization as an intermediate step in the analogical reasoning. But in reality, such an argument is evaluated by identifying the features, that play a central role in the mathematical proof for the source domain, and determining whether analogous features are present, or at least not known to be absent, in the target domain. The underlying idea is to explore the possibility of a common general structure. This can be done even without a clear generalization in mind.

To make the contrast vivid, consider the Rectangles and Boxes example mentioned in section 5.1 (see also Bartha, 2010, pp. 154-5). On the articulation model, the first step is to supply an explicit proof for the result about rectangles. Here is a proof: since perimeter is constant, $x$

$y \quad c$ for some constant; area $x y$ is maximized when $x(c-x)$ is maximized; the First Derivative Test tells us that this happens when $x \quad y$ $c / 2$. Hence, area is maximized for a square. The critical elements in this proof are the formulas for area and perimeter, the assumption that perimeter is constant, and the First Derivative Test. Looking at the target domain (boxes), we see that there are analogous formulas for volume and perimeter, and an analogous theorem about maximizing functions of two variables. Hence, we have a good analogical argument with a plausible conclusion. We don't need to invoke the $n$-dimensional version of the theorem to see this. The evaluation proceeds at the level of facts about the source and target domains, without reference to a general fact of analogy, but the ideal of generalization guides the assessment. Even for this simple example, as noted in (Bartha, 2010), there are subtleties that must be resolved through a consideration of contextual factors. If our objective is to define a logic of analogy, then the articulation model is a failure. But if the goal is to provide guidelines for analogical reasoning, the example counts as a modest success.

Could Norton's account of analogy provide guidance with this example ${ }^{10}$ The material theory tells us to identify and find support for the fact of analogy. This is problematic for two reasons. The first is that we don't need the fact of analogy to do the reasoning. We only arrive at the relevant generalization much later. The second problem is that the material theory provides no help in identifying the fact of analogy.

The deficiencies of the material theory, in terms of providing practical guidance for analogical reasoning, are unsurprisingly linked to its

${ }^{10}$ In fairness, Norton applies the material theory only to empirical science. formal impoverishment. One basic pattern is used for all analogical reasoning. By contrast, the articulation model can provide guidance even without "fully elaborated formal schema". A very similar point applies to exciting work that is taking place in field-specific studies of analogical reasoning in historical sciences such as evolutionary biology and archaeology (Currie, 2016, 2018; Reiss, 2015; Wylie \& Chapman, 2016). The analysis in these studies is domain-specific and aimed at developing practical "toolkits" for assessing analogical arguments. "Local" guidelines, emerging from practice and experience, are broadly consistent with Norton's negative claims, but they are antithetical to the extreme materialism of his positive account. The point is that we can develop useful models for different patterns of analogical reasoning, recognizing that they are capable of refinement.

\section{A theoretical role for quasi-formal theories: justification}

At the end of section 3, I raised the question, "what role can a formal or quasi-formal theory play in the justification of analogical reasoning if it is not an inductive logic?" This section outlines a tentative response on behalf of the articulation model. In brief, the validation of a good analogical argument depends both on a "local" schema and on pragmatic, context-dependent judgments. The articulation model provides a framework that identifies the formal and material elements that figure in the justification of an individual argument, and shows how they work together.

First, we need to understand what it means for the articulation model to validate an analogical argument. The account rests on a set of assumptions. I present them in a highly compressed form here; see (Bartha, 2010) for details.

Assumption 1: we can identify a set of critical factors $X_{1}, \ldots, X_{\mathrm{n}}$ in the source domain.

Referring to Fig. 1 of section 3, let $Q$ be the property of the source domain that is transferred to the target. The prior association is a connection between other features of the source domain and $Q$. The critical factors $X_{1}, \ldots, X_{\mathrm{n}}$ are those features that play an essential role in the prior association. ${ }^{11}$ The articulation model provides models to help identify these critical factors. Since those models are provisional and imprecise, this step is inevitably context-dependent.

Assumption 2: we can identify corresponding features $X_{1}{ }^{*}, \ldots, X_{\mathrm{n}}{ }^{*}$, $Q^{*}$ in the target domain.

This step once again depends upon context. Analogical arguments require us to defend (and criticize) assertions of correspondence.

To establish that we have a good analogical argument, according to the articulation model, we need to show that the same type of connection that holds in the source domain might hold in the target. This is operationalized in terms of a test with two evaluation criteria:

Overlap: there is a nonempty positive analogy. This means that some (at least one) analogues of the critical factors, $X_{1}^{*}, \ldots, X_{\mathrm{n}}^{*}$, are known to hold in the target. ${ }^{12}$

No critical disanalogy: no critical factor belongs to the negative analogy. This means that none of $X_{1} *, \ldots, X_{\mathrm{n}}{ }^{*}$ is known not to hold in the target. $^{13}$

\footnotetext{
11 The features $X_{\mathrm{i}}$ are not shown in Fig. 1, but the idea is that each $X_{\mathrm{i}}$ is either in the positive analogy, the negative analogy or neither (if we don't know whether the analogue $X_{\mathrm{i}}^{*}$ is a feature of the target domain).

${ }^{12}$ In terms of Fig. 1: some of the critical factors are in the positive analogy, P. ${ }^{13}$ In terms of Fig. 1: none of the critical factors belongs to the negative analogy, A \& $\sim$ B.
} 
$\underline{\text { Source } S}$

$\underline{\text { Target } T}$

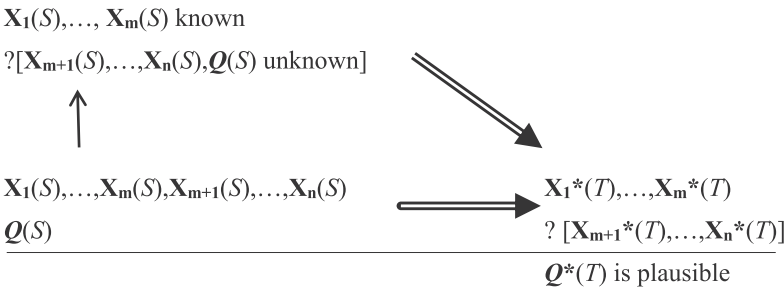

Fig. 3. Justification for an analogical argument.

An analogical argument that satisfies these two criteria has a prima facie plausible conclusion $Q^{*}$. This means that $Q^{*}$ is a serious possibility, i.e., worthy of investigation. According to the articulation model, we have a good analogical argument.

Putting the preceding points together, we see that at the heart of the articulation model, there is, in fact, a general schema for analogical reasoning:

(*) If $X_{1}, \ldots, X_{\mathrm{n}}$ are the critical factors in the source domain and $X_{1}^{*}$, $\ldots, X_{\mathrm{n}}{ }^{*}$ are the target analogues, then the analogical inference (that

$Q^{*}$ is prima facie plausible) is valid if the two conditions Overlap and No critical analogy are satisfied.

This schema is formal and universal, but it is not contextindependent. The schema $(*)$ is stated as a conditional. The antecedent incorporates a context-dependent assessment of critical factors and source-target correspondence. The consequent is a practical recommendation that also needs to be interpreted contextually. In the absence of context, the schema is a lifeless abstraction. Nevertheless, it provides a formal basis for the justification of the analogical argument. That is, we can use it to explain why an analogical argument that satisfies Overlap and No critical analogy has a plausible conclusion.

The key idea is encapsulated in a diagram that is a bit like Fig. 2 (see (Bartha, 2010, p. 272)):

In the diagram, $t_{1}$ represents your actual knowledge situation, consistent with the two assumptions and the assumed satisfaction of the two evaluation criteria. In other words, you know that the critical factors $X_{1}, \ldots, X_{\mathrm{n}}$ hold in the source. You know that at least some of the analogues $X_{1}^{*}, \ldots, X_{\mathrm{m}} *$ hold in the target (Overlap). Finally, you are not aware of any analogues that fail to hold in the target (No critical disanalogy) - that's what we mean by writing "? $\left[\mathbf{X}_{\mathbf{m}} \mathbf{1}^{*}(T), \ldots, \mathbf{X}_{\mathbf{n}} *(T)\right]$ " in the column for the target. Let $t_{0}$ represent a hypothetical "earlier moment" at which you subtract your knowledge of $X_{\mathrm{m}} 1, \ldots, X_{\mathrm{n}}$ and $Q$ $(S)$, and at which you are deciding whether to treat $Q(S)$ as a serious possibility. The moment $t_{0}$ is an artificial construction; there need not have been any such moment. Its introduction allows us to decompose the analogical reasoning into two steps.

The first step (the vertical arrow) is a Reflection argument. So far as your opinion at $t_{0}$ is concerned, you might come to regard $Q(S)$ as a serious possibility at later time $t_{1}$; hence, you should not dismiss it at $t_{0}$. This means that you should regard $Q(\mathrm{~S})$ as a serious possibility at $t_{0}$. The second step (the diagonal arrow) is a Symmetry argument: since there is a perfect analogy (up to critical factors) between the source domain $S$ at $t_{0}$ and the target domain $T$ at $t_{1}$, and you regard $Q$ as plausible in $S$ at $t_{0}$, you should regard $Q^{*}$ as plausible in $T$ at $t_{1}$. That's it!

In just the same way that Fig. 3 is reminiscent of Fig. 2, the above argument echoes Norton's two-step justification. Instead of inductive generalization, the first step (from $S$ at $t_{1}$ to $S$ at $t_{0}$ ) proceeds via Reflection. Instead of deduction, the second step (from $S$ at $t_{0}$ to $T$ at $t_{1}$ ) is based on Symmetry. The complexity of this argument derives from the fact that the driver for the analogical reasoning is the possibility of generalization (rather than a specific generalization, formulated as an intermediate step).

This argument obviously requires clarification and examination. The brief discussion here is meant to establish only three points. First, a formal theory of analogy can fall short as an inductive logic but still play a substantive role in the justification of analogical arguments. Second, the criteria for good analogical reasoning can and should be built right into the argument for justification (this is what happens with the two evaluation criteria). Third, there is a clear contrast with Norton's analysis of justification: analogical inference is justified in terms of potential generalization rather than actual (local) generalization. But Norton's analysis may also be viewed as a special case.

\section{Conclusion}

In his discussion of analogical reasoning, Norton defends the negative thesis that there is no place for formal theories of analogy, as well as a positive account: the material theory. Despite my criticisms, I find the material theory of analogy attractive for its simplicity, its rejection of abstract principles, and its insistence that every analogy is rooted in facts. I have argued that Norton's rejection of all formal principles is based on an unrealistically high standard for a theory of analogical reasoning. It leads to an impoverished positive account that distorts or disqualifies many respectable analogical arguments. A good theory of analogical reasoning needs to provide guidance in evaluating analogical inferences and insight into the justification of such inferences. The articulation model, the target of many of Norton's criticisms, aims to fulfill both objectives for a wider class of analogical arguments than Norton can accommodate.

In section 6, I noted that interesting work is being done in disciplinespecific studies of analogical reasoning. ${ }^{14}$ If Norton could accept "local" or "working" logics of analogy at the disciplinary level, that would parallel his views on other forms of inductive logic. Both those who are sympathetic to Norton's material theory of analogy and those who are sympathetic to formal theories of analogy should explore this idea. Field-specific studies of analogy have great potential as a meeting point for those with different theoretical perspectives.

\section{References}

Aristotle. (1984). In J. Barnes (Ed.), The complete works of Aristotle. Princeton: Princeton University Press.

Bartha, P. (2010). By parallel reasoning: The construction and evaluation of analogical arguments. Oxford: Oxford University Press.

Bartha, P. (2019). In E. N. Zalta (Ed.), "Analogy and analogical reasoning," the stanford encyclopedia of philosophy. Spring 2019 Edition https://plato.stanford.edu/archives /spr2019/entries/reasoning-analogy/.

Carnap, R. (1980). A basic system of inductive logic Part II. In R. C. Jeffrey (Ed.), Studies in inductive logic and probability (Vol. 2, pp. 7-155). Berkeley: University of California Press.

Currie, A. (2016). Ethnographic analogy, the comparative method, and archaeological special pleading. Studies in History and Philosophy of Science, 55, 84-94.

Currie, A. (2018). Rock, bone and ruin. Cambridge, MA: MIT Press.

Dardashti, R., Hartmann, S., Thebault, K., \& Winsberg, E. (2019). Hawking radiation and analogue experiments: A Bayesian analysis. Studies In History and Philosophy of Science Part B: Studies In History and Philosophy of Modern Physics, 67, 1-11.

Dardashti, R., Thebault, K., \& Winsberg, E. (2017). Confirmation via analogue simulation: What dumb holes could tell us about gravity. The British Journal for the Philosophy of Science, 68, 55-89.

Davies, T. R., \& Russell, S. (1987). A logical approach to reasoning by analogy,. In J. McDermott (Ed.), IJCAI 87: Proceedings of the tenth international joint conference on artificial intelligence (pp. 264-270). Los Altos, CA: Morgan Kaufmann.

Fraser, D. (2018). The development of renormalization group methods for particle physics: Formal analogies between classical statistical mechanics and quantum field theory. Synthese. https://doi.org/10.1007/s11229-018-1862-0.

Galilei, G. (1610). The Starry messenger. In Discoveries and opinions of Galileo (pp. 27-58). Garden City, New York: Doubleday Anchor. S. Drake (trans.), 1957.

Hesse, M. B. (1964). Analogy and confirmation theory. Philosophy of Science, 31, 319-327.

Hesse, M. B. (1966). Models and analogies in science. Notre Dame: University of Notre Dame Press.

Hesse, M. B. (1974). The structure of scientific inference. Berkeley: University of California Press.

Joyce, G. H. (1936). Principles of logic ( $3^{\text {rd }}$ ed.). London: Longmans, Green and Co.

\footnotetext{
14 Some of this work is referenced in (Bartha, 2019).
} 
Kuipers, T. (1988). Inductive analogy by similarity and proximity. In D. H. Helman (Ed.), Analogical reasoning: Perspectives of artificial intelligence, cognitive science, and philosophy (pp. 299-313). Dordrecht: Kluwer Academic Publishers, 1988.

Maier, C. L. (1981). The role of spectroscopy in the acceptance of the internally structured atom. New York: Arno Press.

Mill, J. S. (1904). A sytem of logic, ratiocinative and inductive ( $8^{\text {th }}$ ed.). New York: Harper and Bros.

Niiniluoto, I. (1988). Analogy and similarity in scientific reasoning. In D. H. Helman (Ed.), Analogical reasoning: Perspectives of artificial intelligence, cognitive science, and philosophy (pp. 271-298). Dordrecht: Kluwer Academic Publishers, 1988.

Norton, J. (2018). The material Theory of induction, posted version of June 26, 2018. htt ps://www.pitt.edu/ jdnorton/papers/material_theory/material_theory_J une 26_2018_toc.pdf.

Pearl, J. (2009). Causality: Models, reasoning, and inference (2nd ed.). Cambridge: Cambridge University Press.
Poincare, H. (1952). In F. Maitland (Ed.), Science and method. New York: Dover. trans. Polya, G. (1954). Mathematics and plausible reasoning (2 ${ }^{\text {nd }}$ ed.). Princeton: Princeton University Press, 1968, two Vol. s.

Reiss, J. (2015). A pragmatist theory of evidence. Philosophy of Science, 82(3), 341-362. Romeijn, J. W. (2006). Analogical predictions for explicit similarity. Erkenntnis, 64(2), 253-280.

Russell, S. (1986). Analogical and inductive reasoning. PhD thesis. Palo Alto, CA: Stanford University, Department of Computer Science.

Steiner, M. (1998). The applicability of mathematics as a philosophical problem. Cambridge: Harvard University Press.

Sterrett, S. (2006). Models of machines and models of phenomena. International Studies in the Philosophy of Science, 20, 69-80. March.

Wylie, A., \& Chapman, R. (2016). Evidential reasoning in archaeology. Bloomsbury Academic. 